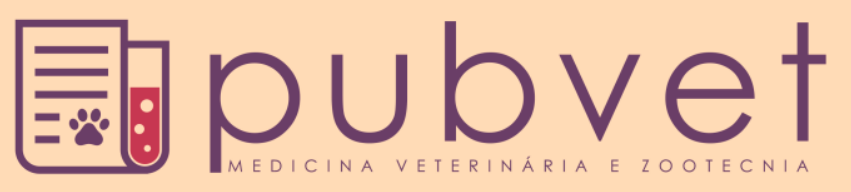

https://doi.org/10.22256/pubvet.v12n2a39.1-7

\title{
Comportamento de bezerras da raça Holandesa alimentadas com silagem pré-secada do capim Tifton 85 (Cynodon spp.)
}

\author{
Wellyngton Tadeu Vilela Carvalho ${ }^{01}$, Daiana Francisca Quirino Villanova ${ }^{02 *}$, \\ Duarte Carvalho Minighin ${ }^{\bullet}$, Wellyson Bougleux Guimarães Lima ${ }^{\bullet}$, Alex de Matos \\ Teixeira ${ }^{\circ}$, Renata Vitarele Gimenes Pereira ${ }^{0}$
}

${ }^{1}$ Professor do Instituto Federal do Sudeste de Minas Gerais - Campus Barbacena. Barbacena-MG Brasil.E-mail: wellyngton.vilela@ifsudestemg.edu.br 2Programa de Pós-Graduação em Zootecnia da Universidade Federal de Viçosa. Viçosa-MG Brasil - E-mail: daiana.q.villanova@ gmail.com 3Programa de Pós-Graduação em Bioengenharia da Universidade Federal de São JoãoDel-Rei. São João Del-Rei-MGBrasil-E-mail:duarteminighin@gmail.com ${ }^{4}$ Engenheiro agrônomo pelo Instituto Federal do Sudeste de Minas Gerais -Campus Barbacena. Barbacena-MG Brasil. E-mail: wellysonlima2013@gmail.com ${ }^{5}$ Professor na Universidade Federal de Uberlândia. Uberlândia-MG Brasil-E-mail: alexmteixeira@yahoo.com.br

${ }^{6}$ Professora do Instituto Federal do Sudeste de Minas Gerais - Campus Barbacena. Barbacena-MG Brasil. E-mail: renata.vitarele@ifsudestemg.edu.br *Autor para correspondência

\begin{abstract}
RESUMO. O uso da silagem pré-secada do capim Tifton 85 (Cynodon spp.) pode ser uma alternativa de volumoso de qualidade para a alimentação animal. Objetivou-se avaliar o comportamento de bezerras da raça Holandesa alimentadas com silagem pré-secada do capim Tifton 85, em substituição à silagem de milho (0,33, 66 e 100\%). Dezesseis animais foram distribuídos em quatro tratamentos e permaneceram em sistema de confinamento durante 21 dias. As avaliações comportamentais foram realizadas nos quatro últimos dias do período experimental durante 24 horas, através da rota de coleta instantânea, com intervalo amostral de 10 minutos e rota de amostragem do tipo scan. As frequências de micção, defecação e ingestão de água também foram registradas. Foi utilizado um delineamento inteiramente casualizado com quatro tratamentos $(0,33$, 66 e $100 \%$ de substituição da silagem de milho por silagem pré-secada do capim Tifton 85) e quatro repetições (número de animais por tratamento). O tempo de ruminação foi influenciado pelas dietas, sendo os maiores valores $(39,76 \%)$ observados para os animais alimentados exclusivamente com silagem de milho. O comportamento dormindo sofreu influência da alimentação variando de 5,81 a 9,28\%, para os níveis de 0 e $66 \%$ de substituição da silagem de milho por silagem pré-secada do capim Tifton 85 , respectivamente. A frequência que os animais beberam água, defecaram e urinaram não apresentou diferença entre os tratamentos. A substituição da silagem de milho pela silagem pré-secada do capim Tifton 85 não provocou alterações no comportamento dos animais no período estudado.
\end{abstract}

Palavras chave: bovino, conservação de volumosos, forragem, ruminante

\section{Holstein heifers' behavior fed with pre-dried silage from Tifton 85 grass (Cynodon spp.)}

ABSTRACT. The use of pre-dried silage from Tifton 85 grass can be a good option of quality forage for animal feeding. The main objective was to evaluate the behavior of the Holstein heifers fed with pre-dried silage, the grass Tifton 85 , replacing the corn silage $(0$, 33, 66 and 100\%). Sixteen animals were distributed in four treatments and remained in feedlot system for 21 days. Behavioral assessments were carried out in the last four days of the trial period during 24 hours, through the instant collection route, with sampling intervals of 10 minutes and type scan sampling route. The frequency of urination, defecation and water intake were also recorded. A completely randomized design with four treatments $(0,33,66$ e $100 \%$ of corn silage replacement for pre-dried grass silage of Tifton 
85), and four repetitions (number of animals per treatments).The rumination time was influenced by diet, being the highest values (39.76\%) observed for animals fed with corn silage. Sleeping behavior influenced food, ranging from 5.81 to $9.28 \%$, to levels of 0 and $66 \%$ corn silage replacement for pre-dried grass silage of Tifton 85 , respectively. The frequency that the animals drank water, defecated and urinated showed no difference among the treatments. The replacement of corn silage by the pre-dried silage of Tifton 85 grass did not cause changes in the behavior of the animals during the studied period.

Keywords: cattle, forage, ruminant, voluminous conservation

\section{Comportamiento de terneras de la raza Holstein alimentadas con silaje pre-secado del pasto Tifton 85 (Cynodon spp.)}

RESUMEN. El uso del silaje pre-secado del pasto Tifton 85 (Cynodon spp.) Puede ser una alternativa de voluminoso de calidad para la alimentación animal. Se evaluó el comportamiento de terneras de la raza Holstein alimentadas con silaje pre-secado del pasto Tifton 85, en sustitución del silaje de maíz (0, 33, 66 y 100\%). Dieciséis animales fueron distribuidos en cuatro tratamientos y permanecieron en sistema de confinamiento durante 21 días. Las evaluaciones comportamentales se realizaron en los cuatro últimos días del período experimental durante 24 horas, por ruta de colecta instantánea, con intervalo de muestra de 10 minutos y ruta de muestreo del tipo scan. Las frecuencias de micción, defecación e ingestión de agua también se registraron. Se utilizó un delineamiento completamente casualizado con cuatro tratamientos $(0,33,66$ y $100 \%$ de sustitución del silaje de maíz por silaje pre-secado del pasto Tifton 85) y cuatro repeticiones (número de animales por tratamiento). El tiempo de rumia fue influenciado por las dietas, siendo los mayores valores $(39,76 \%)$ observados para los animales alimentados exclusivamente con silaje de maíz. El comportamiento durmiendo sufrió influencia de la alimentación variando de 5,81 a $9,28 \%$, para los niveles de 0 y $66 \%$ de sustitución del silaje de maíz por silaje pre-secado del pasto Tifton 85 , respectivamente. La frecuencia que los animales bebieron agua, defecaron y orinaron, no presentó diferencia entre los tratamientos. La sustitución del silaje de maíz por el silaje pre-secado del pasto Tifton 85 , no provocó cambios en el comportamiento de los animales durante el período estudiado.

Palabras clave: bovino, conservación de voluminosos, forraje, rumiante

\section{Introdução}

As pastagens são as principais fontes de manutenção da pecuária brasileira por se tratarem das formas mais baratas de exploração (Reis \& Silva, 2011). Para contornar os problemas gerados pela estacionalidade da produção, métodos para a conservação das forragens podem ser adotados, como a fenação ou a ensilagem, mantendo o fornecimento contínuo de volumoso de qualidade para os animais (Menezes et al., 2009, et al., 2000).

O capim Tifton 85 (Cynodon spp.) vem se destacando por apresentar alta produtividade, facilidade de rebrota e desidratação além de boa aceitabilidade pelos animais, facilidade no manejo, menor custo de produção e resistência a fatores climáticos (Gomes et al., 2015).
No Brasil, o pré-emurchecimento de silagens vem sendo utilizado em regiões de elevada produção leiteira, constituindo um dos métodos mais modernos na conservação de forragens (Evangelista et al., 2004). A produção dos présecados é baseada no emurchecimento parcial das forragens e fermentação microbiana em condições anaeróbicas (Domingues, 2009).

Quando são adotados novos alimentos ou métodos de conservação de silagens no sistema de produção animal, são necessárias pesquisas para possibilitar uma melhor definição do manejo a ser adotado, incrementando a produtividade do rebanho (Prado, 2010). A utilização da silagem pré-secada do capim Tifton 85 (Cynodon spp.) na alimentação de bovinos e, principalmente, em animais jovens, requer estudos dos efeitos benéficos ou adversos provocados pelo consumo e sua influência no comportamento animal (Correia et al., 2012). 
O comportamento animal pode ser afetado pelo tipo de alimento fornecido, por isso estes estudos são importantes ferramentas na avaliação do bemestar animal, possibilitando a identificação de comportamentos anormais e suas possíveis causas, auxiliando na solução de problemas (Broom \& Molento, 2004). O presente trabalho teve por objetivo avaliar o comportamento de bezerras da raça Holandesa alimentadas com silagem présecada de Tifton 85 , em substituição à silagem de milho.

\section{Material e Métodos}

$\mathrm{O}$ experimento foi realizado no município de Barbacena - MG, no Núcleo de Zootecnia, setor de bovinocultura, do Instituto Federal de Educação, Ciência e Tecnologia do Sudeste de Minas Gerais - Campus Barbacena (latitude: $21^{\circ} 13 ' 33^{\prime \prime} \mathrm{S}$, longitude: $43^{\circ} 46^{\prime} 25^{\prime \prime} \mathrm{W}$ e altitude de $1.126 \mathrm{~m})$, em janeiro de 2015 . O clima da região é classificado como tropical de altitude, com temperaturas médias anuais de $18^{\circ} \mathrm{C}$ e precipitação anual média de $1.436 \mathrm{~mm}$ (Köppen \& Geiger, 1928). Dados relacionados à temperatura ambiente e umidade relativa do ar foram coletados durante o período experimental com o auxílio de um termo-higrômetro (Incoterm), instalado no interior das dependências e variaram de 23,2 a $25,8^{\circ} \mathrm{C}$ e 67,7 a $74 \%$, respectivamente. Todos os procedimentos animais foram aprovados pela Comissão de Ética no Uso Animal do Instituto Federal de Educação, Ciência e Tecnologia do Sudeste de Minas Gerais (Protocolo CEUA IFSUDESTEMG n ${ }^{\circ}$ 013/2014).

Utilizou-se 16 bezerras Holandesas com idade média de oito meses e peso médio de $189,3 \mathrm{~kg}$. Os animais foram distribuídos em quatro tratamentos em função da substituição da silagem de milho por silagem pré-secada (Agropecuária Lagartixa) do capim Tifton 85 nos níveis de 0 (tratamento I), 33 (tratamento II), 66 (tratamento III) e 100\% (tratamento IV). O experimento teve a duração de 21 dias. Os dois dias iniciais foram destinados à adaptação dos animais ao tipo de instalação, quinze dias de adaptação às dietas e quatro dias, para coleta dos dados referentes ao comportamento.

As bezerras receberam tratamento contra endo e ectoparasitas antes do período experimental. Os animais foram alojados em camas individuais, em sistema do tipo tie stall dotadas de comedouros e bebedouros individuais, sendo a água fornecida $\mathrm{ad}$ libitum. A dieta foi fornecida duas vezes ao dia
(08:00 e 16:00 horas) (Oliveira et al., 2016). Foi realizada semanalmente a pesagem dos animais, sempre no mesmo horário, para realizar ajustes nas dietas conforme o peso.

$\mathrm{Na}$ tabela 1 encontram-se os dados da composição química da alimentação utilizada. As bezerras foram suplementadas com farelo de soja (46\%), sendo as dietas ajustadas para o ganho médio de $1 \mathrm{~kg} /$ dia, além de considerar $10 \%$ de sobras. Também foi fornecido $30 \mathrm{~g}$ de sal mineral $\left(\mathrm{Guabi}^{\circledR}\right)$ por animal, uma vez ao dia, incorporado ao farelo de soja (NRC, 2001).

Tabela 1. Composição química dos alimentos utilizados na composição das dietas

\begin{tabular}{lccc}
\hline \multirow{2}{*}{ Análises } & \multicolumn{3}{c}{ Amostras } \\
\cline { 2 - 4 } & $\begin{array}{c}\text { Farelo de } \\
\text { soja }\end{array}$ & $\begin{array}{c}\text { Silagem pré- } \\
\text { secada }\end{array}$ & $\begin{array}{c}\text { Silagem de } \\
\text { milho }\end{array}$ \\
\hline Matéria seca (\%) & 87,05 & 39,00 & 40,95 \\
Proteína bruta (\%) & 46,82 & 18,73 & 7,51 \\
Fibra em detergente & 4,53 & 57,89 & 51,48 \\
Extrato etéreo (\%) & 2,51 & 4,10 & 2,64 \\
Cinzas (\%) & 6,86 & 8,74 & 4,64 \\
\hline
\end{tabular}

O consumo total de matéria seca (MS) em $\mathrm{kg}$, o consumo de MS/ $\mathrm{kg}$ de PV (\%) e a relação volumoso:concentrado (\%) dos quatro tratamentos estudados encontram-se na tabela 2.

A avaliação do comportamento dos animais foi realizada durante quatro dias consecutivos, em que os animais foram observados simultaneamente, durante 24 horas ininterruptamente. Foi realizada então a média destes quatro dias de observação, para cada tratamento e variável observada. Foi utilizada rota de coleta instantânea, com intervalo amostral de 10 minutos e rota de amostragem do tipo scan (ou varredura) (Martin et al., 1993) para as seguintes categorias de comportamento: alimentação, ruminação, ócio, dormindo, além de outras atividades.

Tabela 2. Médias do consumo total de matéria seca (CMS) em $\mathrm{kg}$, consumo de MS/ $\mathrm{kg}$ de peso vivo (CMS/ PV) (\%) e a relação volumoso:concentrado (V:C \%) dos tratamentos I ( $100 \%$ de silagem de milho), II (33\% de silagem pré-secada do capim Tifton 85 ), III (66\% de silagem pré-secada do capim Tifton 85$)$ e IV (100\% de silagem pré-secada do capim Tifton 85)

\begin{tabular}{lccc}
\hline Tratamento & CMS (kg) & CMS/\% PV & $\begin{array}{c}\text { Relação V:C } \\
(\%)\end{array}$ \\
\hline I & 6,21 & 3,29 & $79: 21$ \\
II & 5,81 & 3,03 & $77: 23$ \\
III & 5,03 & 2,67 & $83: 17$ \\
IV & 3,99 & 2,12 & $78: 22$ \\
\hline
\end{tabular}


Foi considerada atividade de alimentação quando o animal estava apreendendo o alimento, estando este com a cabeça dentro do cocho (Mitlöhner et al., 2001, Muhammad et al., 2016). Já a ruminação, era a ocorrência de repetidas remastigações dos alimentos, sem apreensão destes. Os animais encontravam-se em ócio (Muhammad et al., 2016), quando estavam parados, em pé (considerado como uma postura ereta inativa, sem nenhuma locomoção) ou deitados (quando o corpo se encontrava em contato com o piso) (Mitlöhner et al., 2001). A atividade dormindo foi considerada quando deitados, com o pescoço virado para o flanco e com os olhos fechados. Foram consideradas como outras atividades, quando o animal interagia com o outro, brincavam ou estavam em estado de atenção, exceto quando estavam ruminando ou se alimentando (Miotto et al., 2014). Durante as observações comportamentais, a frequência que urinavam, defecavam e que consumiam água (quando o animal estava com a cabeça sobre ou no bebedouro) (Mitlöhner et al., 2001), também foi registrada quando estas ocorriam ao final do intervalo amostral.

Foi utilizado um delineamento inteiramente casualizado com quatro tratamentos $(0,33,66 \mathrm{e}$ $100 \%$ de substituição da silagem de milho por silagem pré-secada do capim Tifton 85) e quatro repetições (número de animais por tratamento).

Os dados referentes à porcentagem do tempo (24 horas) que os animais destinaram às atividades de alimentação, ruminação, dormindo, outras atividades e período em ócio, foram submetidos à análise de variância e as médias foram comparadas pelo teste SNK $(\mathrm{P}<0,05)$ através do Sistema de Análises de Variância para Dados Balanceados - SISVAR ${ }^{\circledR}$, Versão 5,0 (Ferreira, 2011).

Para as frequências de ingestão de água, defecar e urinar foram realizados a média do número de ocorrência dos quatro dias de observação, pois alguns parâmetros apresentaram ocorrência zero, devido ao registro da atividade ser realizado quando ocorria ao final do intervalo amostral. Para todos estes parâmetros utilizou-se a análise não paramétrica e teste Kruskal-Wallis para comparação das médias através do programa GrafPad InStat ${ }^{\mathrm{TM}}$, Versão 3.01 (Graphpad software,1998).

\section{Resultados e Discussões}

As dietas fornecidas não influenciaram $(\mathrm{P}>$ $0,05)$ nos tempos destinados à alimentação (Tabela 3), em concordância com os resultados apresentados por Argenta et al. (2017) trabalhando com novilhos confinados alimentados com silagens de sorgo e capim Papuã. E também, com os resultados de Mendonça et al. (2004) os quais avaliaram vacas leiteiras alimentadas com silagem de milho com a relação de volumoso:concentrado de 60:40 e cana de açúcar com as relações de 60:40 e 50:50, que também não encontraram diferenças nos tempos de alimentação em relação às dietas fornecidas.

A média dos quatro tratamentos para a variável tempo de alimentação foi de $15,6 \%$ em $24 \mathrm{~h}$, sendo esta atividade realizada principalmente após o fornecimento das dietas, enquanto o alimento ainda estava fresco. Este comportamento também foi observado no trabalho desenvolvido por Castro et al. (2009) que utilizaram silagem de milho como fonte de volumoso e suplementação com concentrado, assim como no estudo de Segabinazzi et al. (2011), em que os animais consumiram dietas a base de silagem de milho e concentrado na proporção de 40:60, e o restante, e pastagem de milheto. Ambos avaliaram animais em confinamento.

O tempo despendido pelas bezerras para o comportamento ruminando, não diferiu $(P>0,05)$ para os animais que receberam dietas contendo silagem pré-secada do capim Tifton 85 nos três níveis de substituição. Porém, observou-se tempo superior $(\mathrm{P}<0,05)$ de ruminação para os animais alimentados exclusivamente com silagem de milho (Tabela 3), estas diferenças podem ser explicadas devido à qualidade de fibra presente na dieta fornecida. Segundo Bürger et al. (2000), alimentos contendo maiores conteúdos de parede celular aumentam o tempo de ruminação, em concordância com resultados observados no presente estudo. A silagem pré-secada apresentou valores de FDN mais elevados do que a silagem de milho, no entanto, a fibra disponível pode ter sido mais digestível para este alimento quando comparada à silagem de milho.

Mendonça et al. (2004), observaram tempo médio de ruminação de $8,41 \mathrm{~h} /$ dia $(35,04 \%)$ para a dieta de silagem de milho, valor inferior ao tempo médio encontrado neste trabalho, para o mesmo volumoso (Tabela 3). 
As dietas influenciaram $(\mathrm{P}<0,05)$ o tempo em que os animais permaneceram dormindo, sendo que o tratamento com $66 \%$ de silagem pré-secada apresentou o maior valor e as médias dos tratamentos com $33 \%$ e $100 \%$, não diferiram entre si $(\mathrm{P}>0,05)$ (Tabela 3). Em trabalho desenvolvido com novilhas leiteiras alimentadas com subprodutos industriais Castro et al. (2009) descreveram que o tempo do comportamento dormindo, de aproximadamente $4,5 \%$ em $24 \mathrm{~h}$, foi influenciado pelos horários do dia e pelos tratamentos. Este valor é inferior aos descritos neste trabalho, sendo que a interação entre os animais do referido experimento pode ter influenciado neste comportamento, uma vez que foram alojados em grupo.

$O$ comportamento ócio não foi influenciado ( $\mathrm{P}$ $>0,05)$ pelas dietas fornecidas. Pinheiro et al. (2011) avaliando diferentes níveis de inclusão de bagaço de mandioca, encontraram tempos médios diários despendidos com as atividades de ócio de 596 minutos/dia (41,2\% em 24h). Este valor é muito superior ao observado no presente experimento, no entanto estes autores consideraram como ócio o tempo despendido pelos animais dormindo, deitados e em outras atividades.
Tabela 3. Médias do tempo em porcentagem diária (\%), em que as bezerras permaneceram alimentando, ruminando, dormindo, em ócio, e realizando outras atividades, quando alimentadas com $100 \%$ de silagem de milho (I) ou com silagem pré-secada do capim Tifton 85 nas proporções de $33 \%$ (II), $66 \%$ (III) e $100 \%$ (IV)

\begin{tabular}{lccccc}
\hline \multirow{2}{*}{ Tratamento } & \multicolumn{5}{c}{ Comportamento (\%) } \\
\cline { 2 - 6 } & AlimentandoRuminandoDormindo & Ócio & Outros \\
\hline I & 16,75 & $39,76^{\mathrm{a}}$ & $5,81^{\mathrm{b}}$ & 26,56 & 8,02 \\
II & 13,80 & $36,94^{\mathrm{b}}$ & $7,89^{\mathrm{ab}}$ & 29,99 & 8,33 \\
III & 15,20 & $36,02^{\mathrm{b}}$ & $9,28^{\mathrm{a}}$ & 28,55 & 6,94 \\
IV & 16,89 & $33,85^{\mathrm{b}}$ & $7,89^{\mathrm{ab}}$ & 30,77 & 7,55 \\
\hline CV \% & 29,43 & 10,84 & 34,13 & 20,40 & 46,33
\end{tabular}

$\mathrm{CV}$ : coeficiente de variação. Letras minúsculas diferentes na mesma coluna diferem estatisticamente $(\mathrm{P}<0,05)$.

Não houve diferenças nas médias de tempo em que os animais destinaram a outras atividades (Tabela 3). Bürger et al. (2000) em trabalho desenvolvido com animais jovens também não observaram diferenças significativas para 0 mesmo comportamento.

A frequência que os animais beberam água, defecaram e urinaram não apresentou diferenças significativas entre os tratamentos $(\mathrm{P}>0,05)$ (Tabela 4), podendo-se sugerir que os tratamentos não causaram alterações digestivas e urinárias durante o período experimental.

Tabela 4. Médias e medianas da frequência (número de ocorrência em 24 horas) em que as bezerras beberam água, defecaram e urinaram quando foram alimentadas com $100 \%$ de silagem de milho (I) ou com silagem pré-secada do capim Tifton 85 nas proporções de $33 \%$ (II), $66 \%$ (III) e $100 \%$ (IV)

\begin{tabular}{lcccc}
\hline & & \multicolumn{2}{c}{ Parâmetro } \\
\hline & Tratamento & Água & Defecando & Urinando \\
\cline { 2 - 5 } Médias & I & 11,64 & 3,00 & 3,36 \\
& II & 9,78 & 2,64 & 10,14 \\
& III & 10,50 & 10,50 & 6,36 \\
\hline $\mathrm{H}$ & IV & 10,86 & 3,78 & 6,36 \\
\hline P & & 1,27 & 1,97 & 6,28 \\
\hline & & 0,72 & 0,56 & 0,09 \\
Medianas & I & $10,5(3,00-22,5)$ & $3,00(1,50-4,50)$ & $7,80(6,00-9,00)$ \\
& II & $7,50(7,50-16,50)$ & $2,28(0,00-6,00)$ & $3,00(1,50-6,00)$ \\
& III & $9,00(7,50-16,50)$ & $9,00(7,50-16,50)$ & $12,00(3,00-13,50)$ \\
\hline
\end{tabular}

Valores entre parêntesis correspondem aos valores mínimos e máximos.

\section{Conclusão}

A substituição da silagem de milho pela silagem pré-secada do capim Tifton 85, não provocou alterações no comportamento dos animais no período estudado.

Dessa forma, este alimento deve ser testado em relação à sua digestibilidade, parâmetros fisiológicos e outros parâmetros para que possa ser recomendado para utilização nesta espécie e categoria animal.

\section{Agradecimentos}

IF Sudeste MG e Agropecuária Lagartixa. 


\section{Referências Bibliográficas}

Argenta, F. M., Brondani, I. L., Alves Filho, D. C., Nornberg, J. L., Segabinazi, L. R., Restle, J., Weise, M. S., Borchate, D., da Silva Rodrigues, L. \& de Moura, A. F. 2017. Comportamento ingestivo de novilhos alimentados com silagem de capim papuã (Urochloaplan taginea) $\mathrm{x}$ silagem de sorgo (Sorghum bicolor (L.) Moench). Revista Acadêmica: Ciência Animal, 11, 243-253.

Broom, D. M. \& Molento, C. F. M. 2004. Animal welfare: concept and related issues-review. Archives of Veterinary Science, 9, 1-11.

Bürger, P. J., Pereira, J. C., Queiroz, A. C., Coelho, J. F., Agostini, P. S., Valadares Filho, S. C., Cecon, P. R. \& Casali, A. D. P. 2000. Comportamento ingestivo em bezerros holandeses alimentados com dietas contendo diferentes níveis de concentrado. Revista Brasileira de Zootecnia, 29, 236-242.

Castro, K. J., Miranda, N. J. N., Silva, F. A. J., Rocha, C., Fabrícia \& Carneiro , O. R. 2009. Respostas comportamentais de novilhas leiteiras alimentadas com dietas à base de subprodutos agroindustriais. Revista Ciência Agronômica, 40, 306-314.

Correia, B. R., Oliveira, R. L., Jaeger, S. M. P. L., Bagaldo, A. R., Carvalho, G. G. P., Oliveira, G. J. C., Lima, F. H. S. \& Oliveira, P. A. 2012. Comportamento ingestivo e parâmetros fisológicos de novilhos alimentados com tortas do biodiesel. Archivos de zootecnia, 61, 79-89.

Domingues, J. L. 2009. Use of conserved roughage in the horse feeding. Revista Brasileira de Zootecnia, 38, 259-269.

Evangelista, A. R., Peron, A. J. \& Amaral, P. N. C. 2004. Forrageiras não convencionais para silagem-mitos e realidades. Simpósio sobre manejo estratégico da pastagem, 2, 463-507.

Ferreira, D. F. 2011. SISVAR: A Computer Statistical Analysis System. Ciência $e$ Agrotecnologia, 35, 1039-1042.

Gomes, E. P., Rickli, M. E., Cecato, U., Vieira, C. V., Sapias, J. G. \& Sanches, A. C. 2015. Produtividade do capim Tifton 85 sob irrigação e doses de nitrogênio. Revista Brasileira de Engenharia Agrícola e Ambiental, 19, 317323.

Graphpad Software. Versão 3.01. 1998. Graphpad Software, Inc. San Diego, Califórnia.
Köppen, W. \& Geiger, R. 1928. Klimate der Erde. Gotha: Verlag Justus Perthes. Wall-map $150 \mathrm{~cm} \times 200 \mathrm{~cm}$.

Martin, P. R., Bateson, P. P. G. \& Bateson, P. 1993. Measuring behaviour: an introductory guide. Cambridge University Press, Cambridge

Mendonça, S. S., Campos, J. M. S., Valadares Filho, S. C., Valadares, R. F. D., Soares, C. A., Lana, R. P., Queiroz, A. C., Assis, A. J. \& Pereira, M. L. A. 2004. Comportamento ingestivo de vacas leiteiras alimentadas com dietas à base de cana-de-açúcar ou silagem de milho. Revista Brasileira de Zootecnia, 33, 723-728.

Menezes, L. F. G., Segabinazzi, L. R., Brondani, I. L., Restle, J., Arboitte, M. Z., Kuss, F., Pacheco, P. S. \& Rosa, J. R. P. 2009. Silagem de milho e grão de sorgo como suplementos para vacas de descarte terminadas em pastagem cultivada de estação fria. Arquivo Brasileiro de Medicina Veterinária e zootecnia, 61, 182189.

Miotto, F. R. C., Neiva, J. N. M., Restle, J., Silva Falcão, A. J., Castro, K. J. \& Maciel, R. P. 2014. Comportamento ingestivo de tourinhos alimentados com dietas contendo níveis de gérmen de milho integral. Ciência Animal Brasileira, 15, 45-54.

Mitlöhner, F. M., Morrow, J. L., Dailey, J. W., Wilson, S. C., Galyean, M. L., Miller, M. F. \& McGlone, J. J. 2001. Shade and water misting effects on behavior, physiology, performance, and carcass traits of heat-stressed feedlot cattle. Journal of Animal Science, 79, 2327-35.

Muhammad, A. U. R., Xia, C. Q. \& Cao, B. H. 2016. Dietary forage concentration and particle size affect sorting, feeding behaviour, intake and growth of Chinese holstein male calves. Journal of Animal Physiology and Animal Nutrition, 100, 217-223.

Nascimento, J. M., Costa, C., Silveira, A. C. \& Arrigoni, M. D. B. 2000. Influência do método de fenação e tempo de armazenamento sobre a composição bromatológica e ocorrência de fungos no feno de alfafa (Medicago sativa, L. cv. Flórida 77). Revista Brasileira de Zootecnia, 29, 669-677.

NRC. 2001. Nutrient Requirements of Dairy Cattle, 7th rev. edn. Natl. Acad. Press, Washington, DC., Washington.

Oliveira, E. R., Moncao, F. P., Araujo Gabriel, A. M., Santos Abreu, F. S., Moura, L. V., 
Nascimento, F. d. A., Souza, C. V. M. \& Galharte, F. T. A. 2016. Performance and economic analysis of finished lambs in feedlot. Semina: Ciências Agrárias, 37, 293-302.

Pinheiro, A. A., Veloso, C. M., Santana Júnior, H. A., Rocha Neto, A. L., Silva, R. R., Lima, F. B. M., Oliveira, L. N., Azevedo, S. T. \& Cecato, U. 2011. Avaliação dos intervalos e números de observações no comportamento ingestivo de novilhas leiteiras confinadas. Revista Brasileira de Saúde e Produção Animal, 12, 480-490.

Prado, I. N. 2010. Produção de bovinos de corte e qualidade da carne. Eduem, Maringá, Paraná, Brasil.

Reis, R. A. \& Silva, S. C. 2011. Consumo de forragens. In: Berchielli, T. T., Pires, A. V. \& Oliveira, S. G. (eds.) Nutrição de Ruminantes. FUNEP, Jaboticabal, Brazil.
Segabinazzi, L. R., Viégas, J., Freitas, L. S., Brondani, I. L., Argenta, F. M. \& Binotto, J. 2011. Behavior patterns of cows with Charolais or Nellore breed predominance fed diets with plant extract or monensin sodium. Revista Brasileira de zootecnia, 40, 29542962.

Article History:

Received 6 November 2017

Accepted 12 December 2017

Available online 28 January 2018

License information: This is an open-access article distributed under the terms of the Creative Commons Attribution License 4.0, which permits unrestricted use, distribution, and reproduction in any medium, provided the original work is properly cited. 